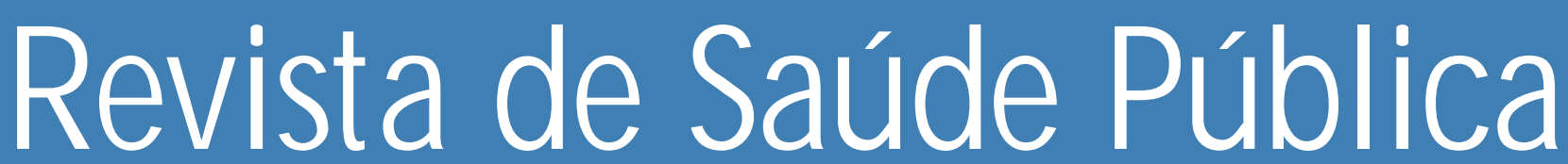

\begin{tabular}{llllllll}
\hline & 0 & $U$ & $R$ & $N$ & $A$ & $L$
\end{tabular}

$0 F$

$\mathbf{P} \mathbf{U}$

H E A L T H

\title{
Análise de concordância entre informações referidas e observadas acerca do estadiamento pubertário entre escolares do sexo feminino
}

\section{Analysis of agreement between self-assessment and observed classification of pubertal development among school girls}

Jael de Paula G uimarães e Afonso D inis Costa Passos

Ambulatório de Atenção à Saúde de Adolescentes da Secretaria Municipal de Saúde de Ribeirão Preto. Ribeirão Preto, SP - Brasil (J.P.G.), Departamento de M edicina Social da Faculdade de Medicina de Ribeirão Preto da Universidade de São Paulo. Ribeirão Preto, SP Brasil (A.D.C.P.) 


\title{
Análise de concordância entre informações referidas e observadas acerca do estadiamento pubertário entre escolares do sexo feminino*
}

\author{
Analysis of agreement between self-assessment and \\ observed classification of pubertal development \\ among school girls
}

\author{
Jael de Paula G uimarães e Afonso D inis Costa Passos \\ Ambulatório de Atenção à Saúde de Adolescentes da Secretaria Municipal de Saúde de Ribeirão \\ Preto. Ribeirão Preto, SP - Brasil (J.P.G.), Departamento de Medicina Social da Faculdade de \\ Medicina de Ribeirão Preto da U niversidade de São Paulo. Ribeirão Preto, SP - Brasil (A.D.C.P.)
}
Resumo
Objetivo Objetivou-se testar a confiabilidade da informação referida acerca do grau de desenvolvimento mamário e dos pêlos pubianos, entre jovens do sexo feminino de diferentes níveis socioeconômicos.

\begin{abstract}
Material e Método
Foram estudadas 445 escolares matriculadas em três estabelecimentos de ensino da cidade de Ribeirão Preto, SP, Brasil. Após observação de fotos dos diferentes estádios de desenvolvimento, segundo padronização de Tanner, as participantes se autoclassificaram em relação aos mesmos. Essas informações foram comparadas com as obtidas por exame físico, realizado imediatamente após a autoclassificação.

Resultados Os percentuais de concordância entre as informações referidas e observadas acerca do desenvolvimento mamário variaram entre $57,3 \%$ e $65,2 \%$. Em relação aos pêlos pubianos, os valores oscilaram entre $65,4 \%$ e $73,5 \%$. Quando considerados simultaneamente, os valores não ultrapassaram 50,0\%. Percentuais elevados de concordância foram observados em estágios correspondentes a etapas críticas do desenvolvimento pubertário, tais como M2, M4 e P4.

Conclusões

Evidencia-se a possibilidade de detecção de etapas significativas do desenvolvimento, como o início da puberdade, a ocorrência da menarca e a desaceleração do final do crescimento estatural. As baixas concordâncias verificadas na análise simultânea das duas variáveis pesquisadas apontam para a necessidade de se aperfeiçoarem metodologias capazes de substituir, com confiabilidade, o exame físico pela auto-avaliação do desenvolvimento pubertário, na realização de estudos de campo.
\end{abstract}

Puberdade. Maturidade sexual. Adolescência.

\footnotetext{
* Parte da dissertação de mestrado de Jael de Paula Guimarães, apresentada ao Departamento de Puericultura e Pediatria da Faculdade de Medicina de Ribeirão Preto - USP, em 1993

Correspondência para/Correspondence to: Afonso D.C. Passos - Departamento de Medicina Social da Faculdade de Medicina de Ribeirão Preto da Universidade de São Paulo. Avenida Bandeirantes, 3900 - 14049-900 Ribeirão Preto, SP - Brasil. E-mail: apassos@ fmrp.usp.br Edição subvencionada pela FAPESP. Processo 96/5999-9.

Recebido em 26.6.1996. Reapresentado em 31.10.1996. Aprovado em 25.11.1996.
} 


\begin{abstract}
Aim To test the reliability of the self-classification concerning levels of breast and pubic hair development among female adolescents of different socioeconomic groups.

Material and Method

Results

Conclusions

A total of 445 female adolescents attending three secondary schools located in Ribeirão Preto, State of S. Paulo, Brazil, were studied. After being familiarised with Tanner's tables of breast and pubic hair development, the adolescents were asked to self classify themselves according to these variables. The results were compared to those obtained by a physical examination carried out soon after.

Agreement rates between referred and observed information on breast development varied from $57.3 \%$ to $65.2 \%$. For pubic hair the values varied from $65.4 \%$ to $73.5 \%$. Total agreement rates for breast and pubic hair development, when considered simultaneously, reached values below $50.0 \%$ in the three schools. However, high agreement rates were observed for some critical degrees, such as $\mathrm{B} 2, \mathrm{~B} 4$ and $\mathrm{PH} 4$.

Significant events, such as the beginning of pubertal development, the menarche and the showing down of the body's development growth could be determined with acceptable levels of agreement. The low agreement rates observed for the simultaneous analysis of the degree of both breast and pubic hair show the need for the perfecting of methodologies that can replace the physical examination of adolescents, once reliable information about pubertal development is obtained in field studies.
\end{abstract}

Puberty. Sex maturation. Adolescence.

\section{INTRO DUÇÃO}

Considera-se como adolescência o período da vida compreendido entre 10 e 20 anos de idade, onde ocorrem rápidas, variadas e amplas transformações nos indivíduos ${ }^{2,8}$. Esta definição, baseada exclusivamente na faixa etária, pode excluir pessoas que já apresentem algum grau de amadurecimento físico antes dos 10 anos de idade, uma vez que o desenvolvimento pubertário, por estar vinculado a fatores genéticos e ambientais, apresenta grande variação no que diz respeito às idades de início e de término, ritmo, intensidade e inter-relação entre os seus fenômenos $^{2,3,6,7}$. Portanto, o estágio de desenvolvimento pubertário representa alternativa muito melhor do que a idade cronológica para se analisar dados relativos ao amadurecimento global dos jovens e para definir com mais precisão as suas patologias.

Para o sexo feminino, as fases do desenvolvimento mamário e dos pelos pubianos constituem parâmetros de exame físico universalmente aceitos na avaliação do grau de amadurecimento pubertário ${ }^{10}$. A possibilidade de se conseguir determinar essas fases mediante uma auto-avaliação das jovens, em substituição ao exame físico tradicional, constitui uma eventualidade que desperta enorme interesse entre os estudiosos dos fenômenos da adolescência, pela grande simplificação que traria para a realização de estudos em comunidade. Para isso, entretanto, torna-se crítica a questão da confiabilidade da informação, a qual tem que ser avaliada criteriosamente antes que pesquisas dessa natureza possam ser levadas adiante.

Como parte de uma investigação acerca do estadiamento pubertário de jovens da cidade de Ribeirão Preto, SP, foi conduzido estudo com vistas a avaliar a qualidade da informação prestada por escolares do sexo feminino acerca do grau de desenvolvimento mamário e de pelos pubianos.

\section{METODOLOGIA}

\section{D escrição das Escolas Estudadas}

Foram estudadas 445 alunas de três escolas de primeiro e segundo graus da cidade de Ribeirão Preto, que serão nomeadas aqui como escolas A, B e C. A escola A é pública e localiza-se em área periférica da região Oeste da cidade. À época da realização do presente estudo (entre setembro e 
dezembro de 1989), o bairro apresentava grande carência de melhoramentos urbanos, com ausência de rede de esgoto, ruas não pavimentadas e deficiências de assistência médica prestada pela única unidade básica de saúde disponível. O comércio local era representado por pequenos estabelecimentos, tipo bares e panificadoras.

A escola $\mathrm{B}$, também pública, localiza-se igualmente na região Oeste, embora bem mais próxima da área central da cidade. A sua infra-estrutura era consideravelmente melhor que a da escola $\mathrm{A}$, com presença de rede de esgoto, ruas pavimentadas e dois postos de saúde num raio de $500 \mathrm{~m}$ da escola. Nessa área localizam-se diversos estabelecimentos comerciais de maior porte, bem como alguns escritórios e consultórios de profissionais liberais.

A escola $\mathrm{C}$, de propriedade privada, localiza-se na região Sul da cidade, em bairro dotado de todos os recursos de uma moderna infra-estrutura urbana. Esta área apresenta uma das mais elevadas valorizações imobiliárias da cidade, sendo ocupada por amplas mansões, agências bancárias, comércio sofisticado e inúmeros escritórios e consultórios de profissionais liberais, incluindo alguns de renome e projeção social.

\section{Critérios de Inclusão}

Ter idade entre 8 anos completos e 18 incompletos no momento de inclusão no estudo.

Ser regularmente matriculada e estar freqüentando a escola no momento da realização do estudo.

Concordar em participar do estudo, no caso de maiores de 16 anos completos.

Concordar em participar do estudo e apresentar um termo de autorização dos pais ou responsáveis, no caso de menores de 16 anos de idade. Nas situações em que os pais ou responsáveis fossem analfabetos, a inclusão se fazia apenas se houvesse o consentimento verbal da parte deles, manifestado pessoalmente à autora da investigação.

\section{O btenção das Informações}

Previamente à obtenção das informações, as participantes receberam explicações detalhadas acerca das diferentes fases do desenvolvimento pubertário. A seguir, em condições de total privacidade e de posse de fotografias dos cinco estágios de desenvolvimento mamário e dos pelos pubianos, adotados e padronizados por Tanner ${ }^{10}$, as participantes foram solicitadas a se autoclassificar em relação a esses estágios, respondendo à seguinte pergunta: "com qual dessas fotografias você acha o seu corpo mais parecido"?

Imediatamente após, procedia-se a exame físico para obtenção do estadiamento de maturação sexual da respectiva aluna, de acordo com a padronização de Tanner ${ }^{10}$. Todas as informações foram obtidas individualmente por uma médica especializada no atendimento a adolescentes, tomando-se o cuidado de que as participantes conhecessem com precisão o significado do que lhes era perguntado.

\section{RESULTAD O S}

Observa-se, pela Tabela 1, que a participação total das alunas atingiu 38,2\%, com valores extremos iguais a $33,7 \%$ e $42,7 \%$, respectivamente nas escolas $\mathrm{A}$ e $\mathrm{C}$. No que diz respeito às diferentes séries, a participação geral variou de $13,3 \%$ a $100,0 \%$. Participações iguais ou superiores a $1 / 3$ das alunas matriculadas foram verificadas em 8 das 11 séries contactadas.

Pela Tabela 2 observa-se que $71,5 \%$ do total das participantes (318/445) se distribuía em idades inferiores a 13 anos, com proporções consideravelmente menores nas faixas etárias mais elevadas.

Tabela 1 - Distribuição das alunas matriculadas e das participantes em cada uma das escolas estudadas, segundo a série escolar a que pertenciam.

Table 1 - Distribution of the participants and the students enrolled in each school by academic year.

\begin{tabular}{|c|c|c|c|c|c|c|c|c|c|c|c|c|c|}
\hline \multirow{3}{*}{\multicolumn{2}{|c|}{$\begin{array}{l}\text { Série } \\
\text { Escolar }\end{array}$}} & \multicolumn{12}{|c|}{ Escola } \\
\hline & & \multicolumn{3}{|c|}{ Escola A } & \multicolumn{3}{|c|}{ Escola B } & \multicolumn{3}{|c|}{ Escola C } & \multicolumn{3}{|c|}{ Total } \\
\hline & & $\begin{array}{l}\text { Matricu- } \\
\text { ladas }\end{array}$ & $\begin{array}{l}\text { Partici- } \\
\text { pantes }\end{array}$ & $\%$ & $\begin{array}{c}\text { M atricu- } \\
\text { ladas }\end{array}$ & $\begin{array}{l}\text { Partici- } \\
\text { pantes }\end{array}$ & $\%$ & $\begin{array}{l}\text { Matricu- } \\
\text { ladas }\end{array}$ & $\begin{array}{l}\text { Partici- } \\
\text { pantes }\end{array}$ & $\%$ & $\begin{array}{c}\text { Matricu- } \\
\text { ladas }\end{array}$ & $\begin{array}{l}\text { Partici- } \\
\text { pantes }\end{array}$ & $\%$ \\
\hline \multirow{8}{*}{$\begin{array}{l}\stackrel{0}{U} \\
\stackrel{U}{U} \\
\stackrel{\circ}{\circ}\end{array}$} & $1^{a}$ & 44 & 7 & 15,9 & 22 & 11 & 50,0 & - & - & - & 66 & 18 & 27,3 \\
\hline & $2^{a}$ & 97 & 48 & 49,5 & 76 & 33 & 43,4 & 32 & 13 & 40,6 & 205 & 94 & 45,9 \\
\hline & 3a & 81 & 28 & 34,6 & 77 & 35 & 45,5 & 34 & 17 & 50,0 & 192 & 80 & 41,7 \\
\hline & $4 \underline{a}$ & 64 & 13 & 20,3 & 67 & 25 & 37,3 & 38 & 25 & 65,8 & 169 & 63 & 37,3 \\
\hline & $5 \underline{a}$ & 46 & 19 & 41,3 & 80 & 36 & 45,0 & 28 & 5 & 17,9 & 154 & 60 & 39,0 \\
\hline & 6a & 30 & 6 & 20,0 & 81 & 27 & 33,3 & 29 & 19 & 65,5 & 140 & 52 & 37,1 \\
\hline & $7 \underline{a}$ & 20 & 11 & 55,0 & 65 & 19 & 29,2 & 18 & 2 & 11,1 & 103 & 32 & 31,1 \\
\hline & $8^{a}$ & 10 & - & - & 55 & 25 & 45,5 & 13 & 1 & 7,7 & 78 & 26 & 33,3 \\
\hline \multirow{3}{*}{\begin{tabular}{l}
$\frac{0}{U}$ \\
\hdashline \\
ol
\end{tabular}} & 10 & - & - & - & 30 & 4 & 13,3 & - & - & - & 30 & 4 & 13,3 \\
\hline & 20 & - & - & - & 21 & 8 & 38,1 & - & - & - & 21 & 8 & 38,1 \\
\hline & 30 & - & - & - & 8 & 8 & 100,0 & - & - & - & 8 & 8 & 100,0 \\
\hline \multicolumn{2}{|c|}{ Total } & 392 & 132 & 33,7 & 582 & 231 & 39,7 & 192 & 82 & 42,7 & 1.166 & 445 & 38,2 \\
\hline
\end{tabular}


Tabela 2 - Distribuição das participantes segundo a idade e escola a que pertenciam.

Table 2 - Distribution of the participants by age and school at which enrolled.

\begin{tabular}{|c|c|c|c|c|c|c|c|c|}
\hline \multirow{4}{*}{$\begin{array}{l}\text { Faixa } \\
\text { Etária }\end{array}$} & \multicolumn{6}{|c|}{ Escola } & \multirow{3}{*}{\multicolumn{2}{|c|}{ Total }} \\
\hline & \multicolumn{2}{|c|}{ A } & \multicolumn{2}{|c|}{$B$} & \multicolumn{2}{|c|}{$C$} & & \\
\hline & & & & intes & & & & \\
\hline & № & $\%$ & № & $\%$ & $\mathrm{~N} N$ & $\%$ & $\mathrm{~N} 0$ & $\%$ \\
\hline $8 \vdash 9$ & 23 & 17,4 & 31 & 13,4 & 12 & 14,6 & 66 & 14,8 \\
\hline $9 \vdash 10$ & 24 & 18,2 & 26 & 11,3 & 17 & 20,7 & 67 & 15,1 \\
\hline $10 \vdash 11$ & 19 & 14,4 & 19 & 8,2 & 22 & 26,8 & 60 & 13,5 \\
\hline $11 \vdash 12$ & 24 & 18,2 & 26 & 11,3 & 8 & 9,8 & 58 & 13,0 \\
\hline $12 \vdash 13$ & 17 & 12,9 & 31 & 13,4 & 19 & 23,2 & 67 & 15,1 \\
\hline $13 \vdash 14$ & 7 & 5,3 & 27 & 11,7 & 3 & 3,7 & 37 & 8,3 \\
\hline $14 \vdash 15$ & 10 & 7,6 & 21 & 9,1 & 1 & 1,2 & 32 & 7,2 \\
\hline $15 \vdash 16$ & 4 & 3,0 & 23 & 10,0 & - & - & 27 & 6,1 \\
\hline $16 \vdash 17$ & 2 & 1,5 & 9 & 3,9 & - & - & 11 & 2,5 \\
\hline $17 \vdash 18$ & 2 & 1,5 & 18 & 7,8 & - & - & 20 & 4,5 \\
\hline Total & 132 & 100,0 & 231 & 100,0 & 82 & 100,0 & 445 & 100,0 \\
\hline
\end{tabular}

A comparação das três escolas no que se refere às concordâncias entre as observações referidas e observadas, dentro de cada estádio de desenvolvimento mamário, é mostrada na Figura 1. Os maiores percentuais de concordância, com valores próximos a $80 \%$, foram verificados nos estádios M1 e M2 na escola A e M4 na escola C. As menores concordâncias, nas três escolas, ocorreram no estádio M3.

A comparação das concordâncias totais entre as distribuições referidas e observadas dos graus de desenvolvimento mamário (Figura 2) revela que as três escolas apresentaram valores próximos, embora aparentemente decrescentes, quando se passa da escola A $(65,2 \%)$ para a B $(63,6 \%)$ e para a C $(57,3 \%)$.

Quando se comparam as três escolas no que diz respeito às concordâncias entre as observações referidas e observadas, em cada estádio de desenvolvimento dos pêlos pubianos (Figura 3), verifica-se que os maiores valores são observados nos estádios P1 (variando entre $80 \%$ e $90 \%$ ) e P4 (entre $73 \%$ e $80 \%$ ). As menores concordâncias são observadas em P3 (entre $25 \%$ e $38,5 \%$ ).

A comparação das concordâncias totais entre as distribuições referidas e observadas dos graus de desenvolvimento dos pêlos pubianos (Figura 4) revela que as escolas A e B apresentaram cifras acima de $70 \%$ ( $73,5 \%$ e $70,2 \%$, respectivamente), caindo para $65,4 \%$ na escola $C$.

As distribuições referidas e observadas dos diferentes estádios de desenvolvimento mamário e dos pêlos pubianos, tomados simultaneamente, são mostradas nas Tabelas 3, 4 e 5, respectivamente, para as escolas A, B e C. As proporções totais de concordância alcançaram valores mínimos de 44,0\% (99/ 225) na escola B e máximos de 48,9\% (64/131) na escola $\mathrm{A}$, passando por um valor intermediário de
Pr a eerresthreis

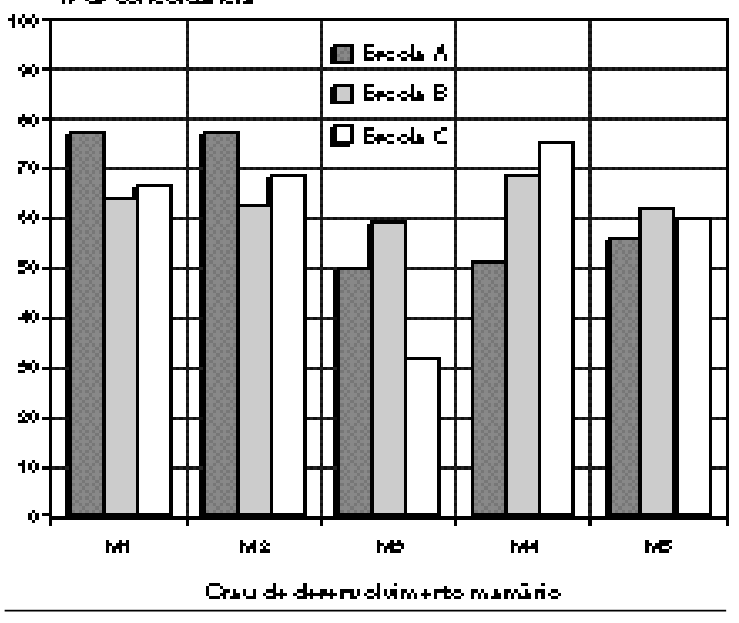

Figura 1 - Proporções de concordância entre as informações referidas e o grau de desenvolvimento mamário observado entre as alunas das escolas A, B e C.

Figure 1 - Proportions of agreement between the referred information and the observed degree of breast development among the students of schools A, B and C.

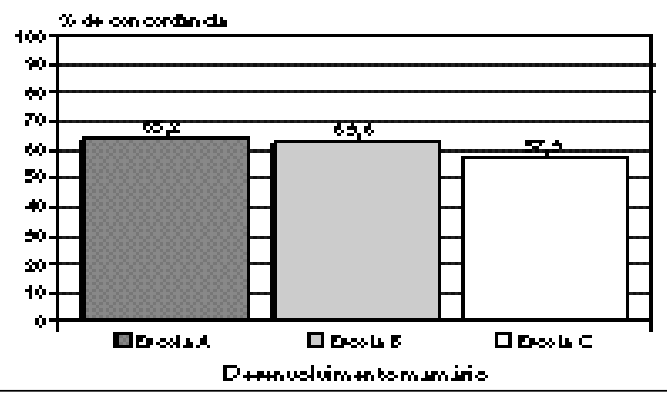

Figura 2 - Proporções totais de concordância entre as informações referidas e o grau de desenvolvimento mamário observado entre as alunas das escolas A, B e C.

Figure 2 - Total proportions of agreement between the referred information and the observed degree of breast development among the students of schools A, B and C. 


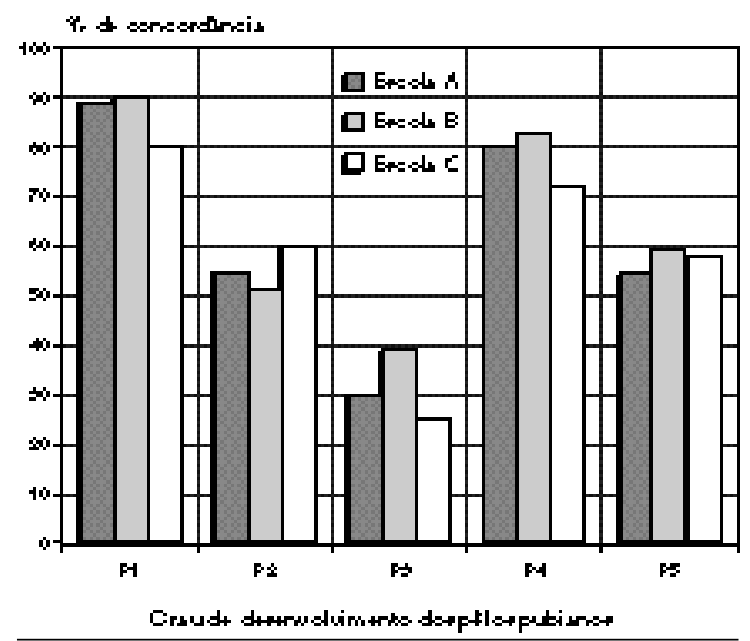

Figura 3 - Proporções de concordância entre as informações referidas e o grau de desenvolvimento dos pêlos pubianos observado entre as alunas das escolas A, B e C.

Figure 3 - Proportions of agreement between the referred information and the observed degree of pubic hair development among the students of schools A, B and C.

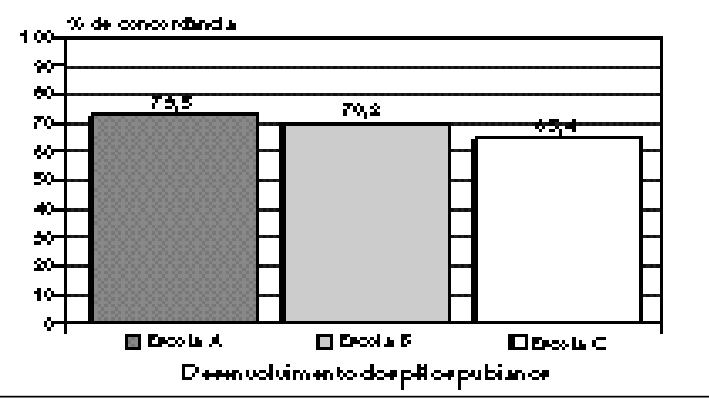

Figura 4 - Proporções totais de concordância entre as informações referidas e o grau de desenvolvimento dos pêlos pubianos observado entre as alunas das escolas A, B e C.

Figure 4 - Total proportions of agreement between the referred information and the observed degree of pubic hair development among the students of schools A, B and C.

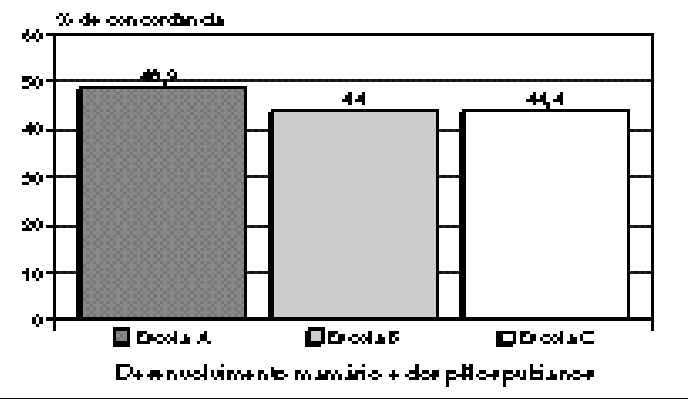

Figura 5 - Proporções totais de concordância entre as informações referidas e o grau de desenvolvimento mamário e dos pêlos pubianos observados entre as alunas das escolas A, B e C.

Figure $\mathbf{5}$ - Total proportions of agreement between the referred information and the observed degree of both breast and pubic hair development among the students of schools $A, B$ and $C$.
44,4\% (36/81) na escola C (Figura 5). As proporções de discordância entre as distribuições referidas e observadas $(51,1 \%, 56,0 \%$ e $55,6 \%$, respectivamente, para as escolas A, B e C), encerram um amplo grau de dispersão em torno da linha diagonal indicativa de concordância entre as distribuições, conforme pode ser visto nas Tabelas 3, 4 e 5 .

\section{DISCUSSÃO}

Os baixos percentuais de participação das estudantes contactadas podem ser explicados por diferentes razões. Em primeiro lugar, as dificuldades referentes ao entendimento da mensagem dirigida aos pais, o que se observou especialmente na escola A, onde o baixo nível socioeconômico das famílias deve ter contribuído para que apenas $1 / 3$ das alunas fossem incluídas no estudo. Recusas dos pais ou das próprias estudantes também se observaram em todas as escolas. $\mathrm{O}$ papel exercido por lideranças naturais dentre as alunas, que ao aceitarem ou recusarem participar do estudo acabavam por influenciar, positiva ou negativamente as suas colegas, fazendo variar a participação nas diferentes classes.

A não-existência de segundo grau na escola $\mathrm{C}$ explica a não-participação de estudantes acima de 15 anos, o que se verifica na escola B. Entretanto, destaca-se a inclusão de alunas nessa faixa etária entre as estudantes da escola A, a qual, a exemplo da C, não mantém segundo grau. Este achado evidencia a ocorrência de um atraso na escolaridade entre algumas estudantes matriculadas na escola A, fenômeno condizente com a pior situação socioeconômica das mesmas.

Os valores relativamente reduzidos de concordância entre as observações referidas e observadas quanto aos estádios de desenvolvimento mamário (Figura 2) e de pelos pubianos (Figura 4) contrastam com resultados obtidos em investigações semelhantes ${ }^{1,4}$, $5,9,11$, onde valores consideravelmente mais elevados foram observados. Uma provável explicação para isso repousa no fato de que tais investigações, quase que invariavelmente, utilizaram outros recursos além das fotos dos estádios de desenvolvimento puberal. Tais recursos incluíram desenhos e/ou textos explicativos capazes de dirimir dúvidas resultantes da simples observação fotográfica. Esses procedimentos foram deliberadamente omitidos na presente investigação, uma vez que se julgou que a adoção dos mesmos poderia induzir a algum viés resultante das diferentes capacidades de leitura e interpretação, certamente existentes entre adolescentes de níveis socioeconômicos distintos. 
Tabela 3 - Distribuição das informações referidas e observadas acerca do grau de desenvolvimento mamário e dos pêlos pubianos entre as alunas da escola $A$.

Table 3 - Distribution of information observed and quoted on the degree of development of breasts and of pubic hair among school-girls of school A.

\begin{tabular}{|c|c|c|c|c|c|c|c|c|c|c|c|c|c|c|c|c|c|c|c|c|c|c|c|c|c|}
\hline $\begin{array}{l}\begin{array}{l}\text { O bser- } \\
\text { vadas } \\
\text { Refe- } \\
\text { ridas }\end{array} \\
\end{array}$ & $\begin{array}{c}M_{1} \\
P_{1}\end{array} \mid$ & $\begin{array}{l}M_{1} \\
P_{2}\end{array}$ & $\begin{array}{c}\mathrm{M} 1 \\
\mathrm{P}_{3}\end{array}$ & $\begin{array}{l}M_{1} \\
P_{4}\end{array}$ & $\begin{array}{l}M_{1} \\
P_{5}\end{array}$ & $\begin{array}{l}M_{2} \\
P_{1}\end{array}$ & $\left|\begin{array}{l}\mathrm{M}_{2} \\
\mathrm{P}_{2}\end{array}\right|$ & $\left|\begin{array}{l}M_{2} \\
P_{3}\end{array}\right|$ & $\begin{array}{c}M_{2} \\
P_{4}\end{array}$ & $\begin{array}{l}M_{2} \\
P_{5}\end{array}$ & $\begin{array}{c}M_{3} \\
P_{1}\end{array}$ & $\begin{array}{c}M_{3} \\
P_{2}\end{array}$ & $\begin{array}{c}M_{3} \\
P_{3}\end{array}$ & & & \begin{tabular}{l|l}
$l_{4}$ & $M$ \\
$P_{1}$ & $P$
\end{tabular} & \begin{tabular}{l|l}
${ }_{4}$ & $M$ \\
${ }_{2}$ & $P_{3}$
\end{tabular} & \begin{tabular}{l|l}
4 & $\mathrm{M}_{4}$ \\
${ }_{3}$ & $\mathrm{P}_{4}$
\end{tabular} & $\begin{array}{l}{ }_{4} \\
M_{4} \\
P_{5}\end{array} \mid$ & $\mid \begin{array}{c}M_{5} \\
P_{1}\end{array}$ & \begin{tabular}{c|c}
$M_{5}$ & $N$ \\
$P_{2}$ &
\end{tabular} & \begin{tabular}{c|c}
$M_{5}$ & $N$ \\
$P_{3}$ & $F$
\end{tabular} & \begin{tabular}{l|l}
$M_{5}$ & $N$ \\
$P_{4}$ & $F$
\end{tabular} & $\begin{array}{c}M_{5} \\
P_{5}\end{array}$ & Total \\
\hline $\mathrm{M}_{1} \mathrm{P}_{1}$ & & 3 & 1 & & & 2 & & & & & & & & & & & & & & & & & & & 35 \\
\hline$M_{1} P_{2}$ & & & 1 & & & & & & & & & 1 & & & & & & 1 & & & & & & & 5 \\
\hline$M_{1} P_{3}$ & 1 & & & & & & & & & & & & & & & & & & & & & & & & 1 \\
\hline $\mathrm{M}_{1} \mathrm{P}_{4}$ & & . & & & & 1 & & & & & & & & & & & & & & & & & & & 3 \\
\hline$M_{1} P_{5}$ & & & & & & & & & & & & & & & & & & & & & & & & & 0 \\
\hline$M_{2} P_{1}$ & 5 & 1 & & & & . & 1 & & & & 5 & & & & 1 & 1 & & & & & & & & & 21 \\
\hline $\mathrm{M}_{2} \mathrm{P}_{2}$ & & & 1 & & & 2 & . & 1 & & & & 2 & & & & 1 & 1 & & & & & & & & 9 \\
\hline $\mathrm{M}_{2} \mathrm{P}_{3}$ & & & & & & & 1 & & & & & & 1 & & & & & 1 & & & & & & & 3 \\
\hline $\mathrm{M}_{2} \mathrm{P}_{4}$ & & & & & & & & & & & & & & & & & & & & & & & & & 1 \\
\hline $\mathrm{M}_{2} \mathrm{P}_{5}$ & & & & & & & & & & & & & & & & & & & & & & & & & 0 \\
\hline$M_{3} P_{1}$ & 2 & 2 & & & & & & & & & & & & & & & & & & & & & & & 5 \\
\hline$M_{3} P_{2}$ & & & & & & & & & & & 1 & & 2 & & & & 1 & & & & & & & & 7 \\
\hline$M_{3} P_{3}$ & & & & & & & & & & & & & . & & & 1 & 1 & & & & & & & & 2 \\
\hline $\mathrm{M}_{3} \mathrm{P}_{4}$ & & & & & & & & & & & & & 1 & & & & & 4 & & & & & & & 7 \\
\hline$M_{3} P_{5}$ & & & & & & & & & & & & & & & & & & & & & & & & & 0 \\
\hline $\mathrm{M}_{4} \mathrm{P}_{1}$ & & & & & & & & & & & & & & & & & & & & & & & & & 0 \\
\hline $\mathrm{M}_{4} \mathrm{P}_{2}$ & & & & & & 1 & 1 & & & & & & & & & & & & & & & & & & 2 \\
\hline $\mathrm{M}_{4} \mathrm{P}_{3}$ & & & & & & & & & & & & & & & & & & 1 & & & & & & & 2 \\
\hline $\mathrm{M}_{4} \mathrm{P}_{4}$ & & & & & & & & & & & & & & & & & & & 2 & & & & 2 & 1 & 11 \\
\hline $\mathrm{M}_{4} \mathrm{P}_{5}$ & & & & & & & & & & & & & & & & & & 1 & & & & & & 2 & 6 \\
\hline$M_{5} P_{1}$ & & & & & & & & & & & 1 & & & & & & & & & & & & & & 1 \\
\hline$M_{5} P_{2}$ & & & & & & & & & & & & & & & & & & & & & & & & & 0 \\
\hline$M_{5} P_{3}$ & & & & & & & & & & & & & & & & & & & & & & & & & 0 \\
\hline $\mathrm{M}_{5} \mathrm{P}_{4}$ & & 1 & & & & & & & & & & & & & & & & 1 & 1 & & & & & 2 & 7 \\
\hline $\mathrm{M}_{5} \mathrm{P}_{5}$ & & & & & & & & & & & & & & & & & & 1 & & & & & & & 3 \\
\hline Total & 37 & 9 & 3 & 2 & 0 & 14 & 5 & 1 & 1 & 0 & 8 & 6 & 4 & 2 & 0 & 12 & 23 & $\begin{array}{ll}3 & 16\end{array}$ & 56 & 0 & 0 & 0 & 4 & 7 & 131 \\
\hline
\end{tabular}

Uma segunda consideração relativa às proporções totais de concordância diz respeito à tendência decrescente observada ao se comparar escolas freqüentadas por alunas progressivamente melhor situadas socioeconomicamente. Muito embora tais diferenças tenham se mostrado reduzidas, a constatação de concordâncias inversamente proporcionais ao padrão socioeconômico sugere ser este achado resultante de uma maior exposição das estudantes mais carentes ao próprio corpo e aos corpos dos familiares. Esta maior exposição se faria em função do uso menos freqüente de roupas íntimas, o que foi constatado inúmeras vezes por ocasião da realização da pesquisa, e do fato de habitarem residências pequenas, muitas vezes sem separação entre os cômodos, o que permitiria maior possibilidade de observação das diferentes fases de desenvolvimento pubertário de familiares do sexo feminino.

A análise de concordância segundo os diferentes estádios de desenvolvimento mamário e de pêlos pubianos mostrou que nas três escolas a maior percentagem de erros ocorreu em relação aos está- 
Tabela 4 - Distribuição das informações referidas e observadas acerca do grau de desenvolvimento mamário e dos pêlos pubianos entre as alunas da escola B.

Table 4 - Distribution of information observed and quoted on the degree of development of breasts and of pubic hair among school-girls of school B.

\begin{tabular}{|c|c|c|c|c|c|c|c|c|c|c|c|c|c|c|c|c|c|c|c|c|c|c|c|c|c|c|}
\hline $\begin{array}{l}\text { O bser- } \\
\text { vadas } \\
\text { Refe- } \\
\text { ridas }\end{array}$ & $\left|\begin{array}{l}M_{1} \\
P_{1}\end{array}\right|$ & $\begin{array}{l}M_{1} \\
P_{2}\end{array}$ & $\begin{array}{c}M 1 \\
P_{3}\end{array}$ & $\begin{array}{l}M_{1} \\
P_{4}\end{array}$ & $\begin{array}{l}M_{1} \\
P_{5}\end{array}$ & $\begin{array}{l}M_{2} \\
P_{1}\end{array}$ & $\begin{array}{l}M_{2} \\
P_{2}\end{array}$ & $\begin{array}{l}M_{2} \\
P_{3}\end{array}$ & $\begin{array}{c}M_{2} \\
P_{4}\end{array}$ & $\begin{array}{l}M_{2} \\
P_{5}\end{array}$ & $\begin{array}{c}M_{3} \\
P_{1}\end{array}$ & $\begin{array}{c}M_{3} \\
P_{2}\end{array}$ & $\begin{array}{c}M_{3} \\
P_{3}\end{array}$ & $\begin{array}{c}M_{3} \\
P_{4}\end{array}$ & $\begin{array}{l}M_{3} \\
P_{5}\end{array}$ & $\begin{array}{c}M_{4} \\
P_{1}\end{array}$ & $\begin{array}{l}M_{4} \\
P_{2}\end{array}$ & $\begin{array}{l}M_{4} \\
P_{3}\end{array}$ & $\begin{array}{c}M_{4} \\
P_{4}\end{array}$ & $\begin{array}{c}M_{4} \\
P_{5}\end{array}$ & $\begin{array}{c}M_{5} \\
P_{1}\end{array} \mid$ & $\begin{array}{c}M_{5} \\
P_{2}\end{array}$ & $\begin{array}{c}M_{5} \\
P_{3}\end{array}$ & $\begin{array}{c}M_{5} \\
P_{4}\end{array}$ & $\begin{array}{c}M_{5} \\
P_{5}\end{array}$ & Total \\
\hline$M_{1} P_{1}$ & 24 & 3 & & & & 5 & 1 & & & & & & & & & & & & & & & & & & & 33 \\
\hline$M_{1} P_{2}$ & 1 & & & & & & 1 & & & & & & & & & & & & & & & & & & & 2 \\
\hline$M_{1} P_{3}$ & 1 & & & & & & & & & & & & & & & & & & & & & & & & & 1 \\
\hline $\mathrm{M}_{1} \mathrm{P}_{4}$ & & & & & & & & & & & & & & & & & & & & & & & & & & 0 \\
\hline$M_{1} P_{5}$ & & . & & & & & & & & & & & & & & & & & & & & & & & & 0 \\
\hline$M_{2} P_{1}$ & 11 & 1 & & & & 7 & 2 & 1 & & & 6 & 2 & & & & & & & & & & & & & & 30 \\
\hline$M_{2} P_{2}$ & & 2 & & & & & 5 & 1 & & & & 2 & 2 & & & & 1 & & & & & & & & & 13 \\
\hline$M_{2} P_{3}$ & & & & & & 1 & 1 & & & & 1 & & & & & & & 1 & & & & & & & & 4 \\
\hline $\mathrm{M}_{2} \mathrm{P}_{4}$ & & & & & & & & & & & & & & 1 & & & & & 1 & & & & & & & 2 \\
\hline$M_{2} P_{5}$ & & & & & & & & & & & & & & & & & & & & & & & & & & 0 \\
\hline$M_{3} P_{1}$ & 1 & . & & & & 1 & & & & & 2 & & & & & & & & & & & & & & & 4 \\
\hline$M_{3} P_{2}$ & & 1 & & & & & 1 & & & & & 4 & & & & & & 1 & & & & & & & & 7 \\
\hline$M_{3} P_{3}$ & & & & & & 1 & & & & & 1 & 5 & 1 & & & & & 1 & 1 & & & & & & & 10 \\
\hline$M_{3} P_{4}$ & & & & & & & & & & & & 1 & & 8 & 1 & & & 1 & 3 & & & & & 1 & & 15 \\
\hline$M_{3} P_{5}$ & & & & & & & & & & & & & & & & & & & & 1 & & & & & 1 & 2 \\
\hline$M_{4} P_{1}$ & & & & & & & & & & & & & & & & & & & & & & & & & & 0 \\
\hline$M_{4} P_{2}$ & & & & & & & & & & & & & & & & & & 1 & & & & & & & & 1 \\
\hline$M_{4} P_{3}$ & & & & & & & & & & & & & 1 & & & & & 1 & & & & & & & & 2 \\
\hline $\mathrm{M}_{4} \mathrm{P}_{4}$ & & & & & & & & & & & & & 1 & & & & & & 8 & 13 & & & & 2 & 7 & 31 \\
\hline $\mathrm{M}_{4} \mathrm{P}_{5}$ & & & & & & & & & & & & & & & & & & & 2 & 9 & & & & 2 & 9 & 22 \\
\hline$M_{5} P_{1}$ & & & & & & 1 & & & & & & & & & & & & & & & & & & & & 1 \\
\hline$M_{5} P_{2}$ & & & & & & & & & & & & & & & & & & & & & & & & & 1 & 1 \\
\hline$M_{5} P_{3}$ & & & & & & & & & & & & & & & & & & & & & & & & & & 0 \\
\hline $\mathrm{M}_{5} \mathrm{P}_{4}$ & & & & & & & & & & & & & & & & & & & & 1 & & & & 10 & 8 & 19 \\
\hline$M_{5} P_{5}$ & & & & & & & & & & & & & & & & & & & 1 & 3 & & & & 1 & 20 & 25 \\
\hline Total & 38 & 7 & 0 & 0 & 0 & 16 & 11 & 2 & 0 & 0 & 10 & 14 & 5 & 9 & 1 & 0 & 1 & 6 & 16 & 27 & 0 & 0 & 0 & 16 & 46 & 225 \\
\hline
\end{tabular}

gios intermediários, respectivamente M3 e P3 (Figuras 1 e 3 ). Isso reflete, muito possivelmente, as dificuldades de reconhecimento de uma fase menos marcada nas suas características individuais e sujeita, portanto, a ser mais facilmente confundida.

Por outro lado, foram observados percentuais elevados de concordância em alguns estágios indicativos de momentos críticos do desenvolvimento pubertário, mormente M2, M4 e P4. Assim, apesar das limitações, verifica-se a possibilidade de detecção de etapas significativas do desenvolvimento, tais como o início da puberdade, a ocorrência da menarca e o término do desenvolvimento estatural.

Se as proporções de concordância entre as informações referidas e observadas apresentaram alguns valores aquém do desejado, quando se consideraram isoladamente os graus de desenvolvimento mamário e dos pelos pubianos, seria de se antever que estas concordâncias viessem a mostrar percentuais ainda mais baixos ao se proceder o estudo destas duas variáveis, medidas simultaneamente. Com efeito, nas três escolas foram verificadas grandes variações en- 
Tabela 5 - Distribuição das informações referidas e observadas acerca do grau de desenvolvimento mamário e dos pêlos pubianos entre as alunas da escola $\mathrm{C}$.

Table 5 - Distribution of information observed and quoted on the degree of development of breasts and of pubic hair among school-girls of school C.

\begin{tabular}{|c|c|c|c|c|c|c|c|c|c|c|c|c|c|c|c|c|c|c|c|c|c|c|c|c|c|c|}
\hline $\begin{array}{l}\text { O bser- } \\
\text { vadas } \\
\text { Refe- } \\
\text { ridas }\end{array}$ & $\begin{array}{l}M_{1} \\
P_{1}\end{array}$ & $\begin{array}{c}M_{1} \\
P_{2}\end{array}$ & $\begin{array}{l}M 1 \\
P_{3}\end{array}$ & $\begin{array}{l}M_{1} \\
P_{4}\end{array}$ & $\begin{array}{c}M_{1} \\
P_{5}\end{array} \mid$ & $\begin{array}{c}M_{2} \\
P_{1}\end{array}$ & $\begin{array}{l}M_{2} \\
P_{2}\end{array}$ & $\begin{array}{c}M_{2} \\
P_{3}\end{array}$ & $\begin{array}{c}M_{2} \\
P_{4}\end{array}$ & $\begin{array}{l}M_{2} \\
P_{5}\end{array}$ & $\begin{array}{c}M_{3} \\
P_{1}\end{array}$ & $\begin{array}{c}M_{3} \\
P_{2}\end{array}$ & $\begin{array}{c}M_{3} \\
P_{3}\end{array}$ & $\begin{array}{l}M_{3} \\
P_{4}\end{array}$ & $\begin{array}{c}M_{3} \\
P_{5}\end{array}$ & $\begin{array}{c}M_{4} \\
P_{1}\end{array}$ & $\begin{array}{c}M_{4} \\
P_{2}\end{array}$ & \begin{tabular}{l|l}
$M_{4}$ & $M$ \\
$P_{3}$ & $P$
\end{tabular} & $\begin{array}{l}M_{4} \\
P_{4}\end{array}$ & $\begin{array}{ll}M_{4} & \\
P_{5} & \end{array}$ & \begin{tabular}{l|l}
$M_{5}$ & 1 \\
$P_{1}$ &
\end{tabular} & \begin{tabular}{c|c}
$\mathrm{M}_{5}$ & $\mathrm{M}$ \\
$\mathrm{P}_{2}$ & $\mathrm{P}$
\end{tabular} & \begin{tabular}{l|l}
$\mathrm{M}_{5}$ & $\mathrm{~N}$ \\
$\mathrm{P}_{3}$ & $\mathrm{~F}$
\end{tabular} & \begin{tabular}{c|c}
$\mathrm{M}_{5}$ & $\mathrm{M}$ \\
$\mathrm{P}_{4}$ & $\mathrm{P}$
\end{tabular} & $\begin{array}{l}M_{5} \\
P_{5}\end{array}$ & Total \\
\hline$M_{1} P_{1}$ & 12 & . & & & & 2 & 2 & & & & & 1 & & & & & & & & & & & & & & 17 \\
\hline$M_{1} P_{2}$ & 1 & 2 & & & & & & & & & & & & & & & & & & & & & & & & 3 \\
\hline$M_{1} P_{3}$ & & 1 & & & & & & & & & & & & & & & & & & & & & & & & 1 \\
\hline $\mathrm{M}_{1} \mathrm{P}_{4}$ & & & & & & & & & & & & & & & & & & & & & & & & & & 0 \\
\hline$M_{1} P_{5}$ & & & & & & & & & & & & & & & & & & & & & & & & & & 0 \\
\hline $\mathrm{M}_{2} \mathrm{P}_{1}$ & 2 & . & & & & 5 & 1 & & & & 2 & 1 & & & & & & & & & & & & & & 11 \\
\hline $\mathrm{M}_{2} \mathrm{P}_{2}$ & 1 & 3 & & & & & 4 & & & & 1 & 2 & 3 & & & & & & & & & & & & & 14 \\
\hline$M_{2} P_{3}$ & & 2 & & & & & & & & & & 1 & & & & & & & & & & & & & & 3 \\
\hline $\mathrm{M}_{2} \mathrm{P}_{4}$ & & & & & & & & & & & & & & & & & & & 1 & & & & & & & 1 \\
\hline$M_{2} P_{5}$ & & & & & & & & 1 & & & & & & & & & & & & & & & & & & 1 \\
\hline $\mathrm{M}_{3} \mathrm{P}_{1}$ & & & & & & & . & & & & 1 & & & & & & & & & & & & & & & 1 \\
\hline$M_{3} P_{2}$ & & & & & & & 1 & & & & 1 & 3 & & & & & & & & & & & & & & 5 \\
\hline$M_{3} P_{3}$ & & & & & & & & & & & 1 & 1 & 1 & & & & & & & & & & & & & 3 \\
\hline $\mathrm{M}_{3} \mathrm{P}_{4}$ & & & & & & & & & & & & & 2 & & & & & & 2 & & & & & & & 4 \\
\hline$M_{3} P_{5}$ & & & & & & & & & & & & & & & & & & & & & & & & & & 0 \\
\hline $\mathrm{M}_{4} \mathrm{P}_{1}$ & & & & & & & & & & & & & & & & & & & & & & & & & & 0 \\
\hline $\mathrm{M}_{4} \mathrm{P}_{2}$ & & & & & & & & & & & & & & & & & & & & & & & & & & 0 \\
\hline $\mathrm{M}_{4} \mathrm{P}_{3}$ & & & & & & & & & & & & & 1 & 1 & & & & & 1 & & & & & & & 3 \\
\hline $\mathrm{M}_{4} \mathrm{P}_{4}$ & & & & & & & & & & & 1 & & & & & & & & 4 & 1 & & & & & 1 & 7 \\
\hline $\mathrm{M}_{4} \mathrm{P}_{5}$ & & & & & & & & & & & & & & & & & & & 1 & 2 & & & & & 1 & 4 \\
\hline$M_{5} P_{1}$ & & & & & & & & & & & & & & & & & & & & & & & & & & 0 \\
\hline$M_{5} P_{2}$ & & & & & & & & & & & & & & & & & & & & & & & & & & 0 \\
\hline$M_{5} P_{3}$ & & & & & & & & & & & & & & & & & & & & & & & & & & 0 \\
\hline $\mathrm{M}_{5} \mathrm{P}_{4}$ & & & & & & & & & & & & & & & & & & & & & & & & \begin{tabular}{l|l}
1 & 1 \\
\end{tabular} & 1 & 2 \\
\hline$M_{5} P_{5}$ & & & & & & & & & & & & & & & & & & & & & & & & & 1 & 1 \\
\hline Total & 16 & 8 & 0 & 0 & 0 & 7 & 8 & 1 & 0 & 0 & 7 & 9 & 7 & 1 & 0 & 0 & 0 & 0 & 9 & 3 & 0 & $\begin{array}{ll}0 & 0\end{array}$ & 0 & \begin{tabular}{l|l}
1 & 4
\end{tabular} & 4 & 81 \\
\hline
\end{tabular}

tre as autoclassificações e as medidas consideradas padrão, resultando que em nenhuma delas a concordância total atingiu sequer a cifra de 50\% (Tabelas 3, 4 e 5). Esses baixos percentuais apresentam-se em oposição aos valores superiores a $80 \%$ obtidos em duas investigações semelhantes, levadas a efeito em São Paulo e nos Estados Unidos ${ }^{1,9}$. Na primeira, além do pequeno número de participantes (42), a falta de um adequado detalhamento metodológico dificulta a comparação com os achados da presente investigação. Na segunda, vale dizer que as fotografias dos diferentes estádios foram substituídas por desenhos detalhados e notas explicativas, o que deve ter contribuído para aumentar a margem de "acertos" das participantes.

A utilização rotineira da auto-avaliação em estudos envolvendo comunidades, em substituição ao exame físico, seria altamente desejável. Isto viria a simplificar enormemente a realização de tais estudos e reduziria de maneira acentuada a proporção de recusas, uma vez que eliminaria as dificuldades decorrentes da exposição a um exame certamente cons- 
trangedor, numa situação em que os potenciais participantes não sentem qualquer necessidade de realização dos mesmos.

Entretanto, os resultados da presente investigação, particularmente no que diz respeito à confiabilidade da informação referente à análise simultânea dos estádios de desenvolvimento da mama e dos pelos pubianos, apontam para a existência de limitações do uso da autoclassificação baseada na simples observação das figuras-padrões. Mesmo os trabalhos que referem resultados mais promissores, provavelmente em decorrência do uso de recursos adicionais à observação de fotos, registram percentuais de discordância que oscilam entre $10 \%$ e $20 \%$, cifras potencialmente capazes de comprometer a qualidade das conclusões. Conforme mencionado anteriormente, a adoção, em situações similares à do presente trabalho, de alguns dos procedimentos usados em outros estudos com vistas a melhorar a confiabilidade das informações, poderia ter como conseqüência a introdução de vieses devidos às diferenças dos subconjuntos de populações estudadas. Em vista disso, e face à relevância de se conseguir alternativas confiáveis à substituição de exames físicos, delineiase a necessidade de serem aperfeiçoadas metodologias capazes de elevar o nível de confiabilidade de

\section{REFERÊNCIAS BIBLIOG RÁFICAS}

1. BROOKS-GUNN, J.; WARREN, M. P.; ROSSO, J.; GARGIULO, J. Validity of self-report measures of girls'pubertal status. Child Dev., 58: 829-41, 1987.

2. COLLI, A. S. Maduración sexual de los adolescentes de São Paulo. In: Organizacion Panamericana de la Salud. La salud del adolescente y del joven en las Américas. Washington, D.C., 1985. p. 249-258. (OPS - Publ. Cient., 489).

3. COLLI, A. S. Maturação sexual na população brasileira: limites de idade. J. Pediatr. (S. Paulo), 60: 173-5, 1986.

4. DUKE, P. M.; LITT, I. F.; GROSS, R. T. Adolescents' selfassessment of sexual maturation. Pediatrics, 66: 918-20, 1980.

5. KOZINETS, C. A. Two noninvasive methods to measure female maturation. J. Sch. Health, 56: 440-2, 1986.

6. MARSHAL, W. A. \& TANNER, J. M. Variations in pattern of pubertal changes in girls. Arch. Dis. Child., 44: 291-303, 1969. estudos desta natureza, no Brasil, minimizando os riscos de introdução de vieses.

Possíveis alternativas poderiam, talvez, incluir explicações prévias exaustivas acerca dos diferentes graus de desenvolvimento pubertário, utilizando-se de recursos como diapositivos, desenhos ou filmes e envolvendo a participação ativa de professores que tenham contacto próximo com os alunos. Ou então, propiciar a oportunidade de que as participantes tenham acesso prévio a fotos e/ou desenhos detalhados, eventualmente levando-os consigo para casa no dia anterior à obtenção das informações. Assim, poderiam analisar detidamente o seu próprio corpo, compará-lo com as figuras-padrões e enquadrá-lo no estádio que melhor corresponde ao seu desenvolvimento.

Pela importância de que se reveste o assunto, estas e outras alternativas metodológicas que venham a ser pensadas merecem ser investigadas cuidadosamente, visando conseguir um modo de determinação do grau de desenvolvimento pubertário que reúna características de elevada simplicidade, aceitabilidade e confiabilidade. Somente assim estudos amplos poderão ser viabilizados com freqüência, fornecendo dados mais precisos e atuais acerca deste importante fenômeno do desenvolvimento humano.

7. OJEDA, E. N. S. et al. Adolescencia y juventud: aspectos demográficos y epidemiológicos. In: Organizacion Panamericana de la Salud. La salud del adolescente y del joven en las Americas. Washington, D.C., 1985. p. 3-19. (OPS -Publ. Cient., 489).

8. ORGANIZACION MUNDIAL DE LA SALUD. Problemas de salud de la adolescencia. Ginebra, 1965. (OMS. Série de Informes Técnicos, n. 308).

9. SAITO, M. I. Maturação sexual: auto-avaliação do adolescente. Pediatria (S. Paulo), 6: 111-5, 1984.

10. TANNER, J. M. The development of the reproductive system. In: Growth at adolescence. 2nd ed., Oxford, Blackwell Scientific Publications, 1962.

11.WILLIANS, R.L. et al. Adolescent self-assessment of sexual maturation. Effects of fatness classification and actual sexual maturation stage. J. Adolesc. Health Care, 9: 480-2, 1988. 